\title{
Comparative Study of Input Shaping with Different Polarities and Modes Selection in Hybrid Control Schemes of a DPTOC System
}

\author{
M.Z. Mohd Tumari, M.S. Saealal, K.H. Ghazali and M.R. Ghazali \\ Faculty of Electrical and Electronics Engineering, Universiti Malaysia Pahang, \\ 26600 Pekan, Pahang, Malaysia
}

\begin{abstract}
This study presents a comparative assessment on input shaping techniques with different polarities and sways frequency modes in hybrid control schemes of a Double-Pendulum-Type Overhead Crane (DPTOC) System. The hybrid control schemes consist of cart position trajectory tracking and sway control of a DPTOC System. A non-linear DPTOC System is considered and the dynamic model of the system is derived using the Euler-Lagrange formulation. The proposed method known as the Single Input Fuzzy Logic Controller (SIFLC) reduces the Conventional two-input FLC (CFLC) to a Single Input Single Output (SISO) controller. The SIFLC is developed for position control of cart movement. This is then extended to incorporate positive and negative input shaping schemes for both hook and load sway angle suppression. Both positive and negative input shaping with different modes selection is designed based on the properties of the system. The results of the response with the controllers are presented in time and frequency domains. The performances of control schemes are examined in terms of level of input tracking capability, sway angle reduction and time response specifications in comparison to SIFLC controller. Finally, a comparative assessment of the control techniques is discussed and presented.
\end{abstract}

Key words: Double-pendulum-type-overhead-crane, sway control, trajectory tracking, single input fuzzy and input shaping, cart movement, Malaysia

\section{INTRODUCTION}

The main objective of controlling a crane system is transporting the load as fast as possible without causing any excessive swing at the final position. However, most of the common crane results in a swing motion when payload is suddenly stopped after a fast motion. Various attempts in controlling cranes system based on open- and closed-loop control system have been proposed. For example, open loop time optimal strategies were applied to the crane by many researchers (Manson, 1992; Auernig and Troger, 1987). Poor results were obtained in these studies because open-loop strategy is sensitive to the system parameters and could not compensate for the effect of wind disturbance. In other hand, feedback control which is well known to be less sensitive to disturbances and parameter variations has also been adopted for controlling the crane system. For example, PD controllers has been proposed for both position and anti-swing controls (Omar, 2003). However, the performance of the controller is not very effective in eliminating the steady state error. In addition, an adaptive control strategy has also been proposed by Yang and
Yang (2007). However, the control technique requires a non-linear control theory which needs a complicated mathematical analysis. The modern control approaches include Fuzzy Logic Controller (FLC) has also been proposed for controlling the crane system by several researchers (Lee and Cho, 2001). Although, those modern control methods are very promising for DPTOC applications, they require substantial computational power because of complex decision making processes. However, it is possible to take full advantages of FLC for DPTOC application if the computational time of FLC is minimized. In this study, the Single Input Fuzzy Controller (SIFLC) is proposed. The SIFLC is a simplification of the Conventional Fuzzy Controller (CFLC). It is achieved by applying the Signed Distance Method (Choi et al., 2000) where the input to SIFLC is only one variable known as distance. This is in contrast to the CFLC which requires an error and the derivative (change) of the error as its inputs. The reduction in the number of inputs simplifies the rule table to one-dimensional, allowing it to be treated as a Single Input Single Output (SISO) controller. As SIFLC can be treated as SISO controller, it can be a practical controller for DPTOC System. As the objective

Corresponding Author: M.Z. Mohd Tumari, Faculty of Electrical and Electronics Engineering, Universiti Malaysia Pahang, 26600 Pekan, Pahang, Malaysia 
of the controlling crane system is to transfer a load from one location to another location, the position error and the velocity of the cart will be the input of the SIFLC. However, the SIFLC is limited for position control of cart and cannot cater for sway control. To overcome this problem, an input shaping schemes is incorporated to the system to suppress the sway of hook and load angle, especially when the cart reaches the desired position (Ahmad et al., 2009a). In previous research, input shaping schemes has been proposed for sway angle suppression of various types of crane system (Ahmad et al., $2009 \mathrm{~b}, \mathrm{c}, 2010$ ). Moreover, the previous study also proved that the positive input shaper with high robustness increases the delay in the cart position responses (Ahmad et al., 2009d, e). To reduce the delay in the cart position response, negative amplitude input shapers have been introduced and investigated. By allowing the shaper to contain negative impulses, the shaper duration can be shortened while satisfying the same robustness constraint. However, the proposed input shaping with different polarities from previous research only limited to a single sway motion of a gantry crane system.

In this study, the effectiveness of the Proposed Hybrid Control Method as well as the differences in polarity and variety in mode selection for input shaper is evaluated to a nonlinear DPTOC Model. Control strategies based on SIFLC with positive and negative input shaper controller are investigated. A simulation environment is developed within Simulink and Matlab for evaluation of performance of the control schemes. Simulation results of the response of the DPTOC System with the controllers are presented in time and frequency domains. The performances of the control schemes are examined in terms of level of input tracking capability, swing angle reduction and time response specifications in comparison to the SIFLC control. Finally, a comparative assessment of the control techniques is presented and discussed.

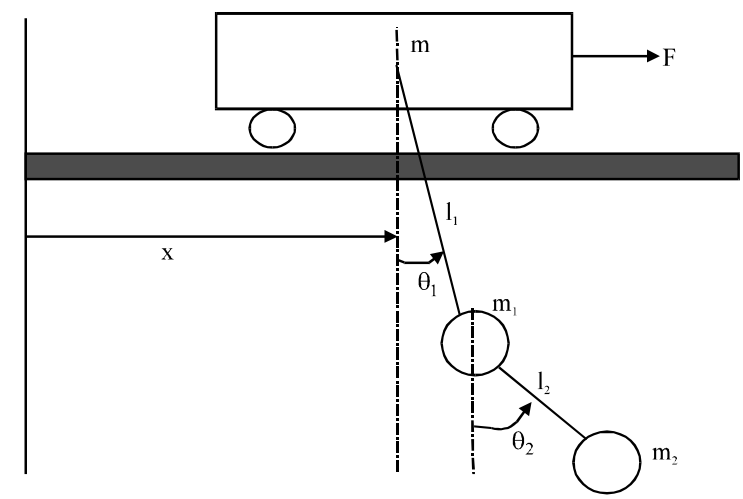

Fig. 1: Description of the DPTOC System

\section{MATERIALS AND METHODS}

The DPTOC System with its hook and load considered in this research is shown in Fig. 1 where $\mathrm{x}$ is the cart position, $m$ is the cart mass and $m_{1}$ and $m_{2}$ are the hook and load mass, respectively. $\theta_{1}$ is the hook swing angle, $\theta_{2}$ is the load swing angle, $l_{1}$ and $l_{2}$ are the cable length of the hook and load, respectively and $F$ is the cart drive force. In this simulation, the hook and load can be considered as point masses. This study provides a brief description on the modeling of the DPTOC System as a basis of a simulation environment for development and assessment of the composite control techniques. The Euler-Lagrange formulation is considered in characterizing the dynamic behavior of the crane system incorporate payload. By Lagrange's equations, the dynamic model of the DPTOC System shown in Fig. 1 is assumed to have the following form (Spong, 1998):

$$
M(q) \ddot{q}+C(q, \dot{q}) \dot{q}+G(q)=\bar{\tau}
$$

Where, the matrices $M(q) \in \Re^{3 \times 3}, C(q, \dot{q}) \in \Re^{3 \times 3}$ and $G$ (q) $€ \Re^{3}$ represent the inertia, Centrifugal-Coriolis terms and gravity, respectively and are defined as:

$$
\begin{gathered}
M(q)=\left[\begin{array}{ccc}
m+m_{1}+m_{2} & \left(m_{1}+m_{2}\right) l_{1} \cos \theta_{1} & m_{2} 1_{2} \cos \theta_{2} \\
\left(m_{1}+m_{2}\right) l_{1} \cos \theta_{1} & \left(m_{1}+m_{2}\right) l_{1}^{2} & m_{2} 1_{1} 1_{2} \cos \left(\theta_{1}-\theta_{2}\right) \\
m_{2} l_{2} \cos \theta_{2} & m_{2} 1_{1} l_{2} \cos \left(\theta_{1}-\theta_{2}\right) & m_{2} 1_{2}^{2}
\end{array}\right] \\
C(q, \dot{q})=\left[\begin{array}{ccc}
0 & -\left(m_{1}+m_{2}\right) 1_{1} \dot{\theta}_{1} \sin \theta_{1} & -m_{2} 1_{2} \dot{\theta}_{2} \sin \theta_{2} \\
0 & 0 & m_{2} 1_{1} 1_{2} \dot{\theta}_{1} \sin \left(\theta_{1}-\theta_{2}\right) \\
0 & -m_{2} 1_{1} 1_{2} \dot{\theta}_{1} \sin \left(\theta_{1}-\theta_{2}\right) & 0
\end{array}\right] \\
G(q)=\left[\begin{array}{lll}
0 & \left(m_{1}+m_{2}\right) g 1_{1} \sin \theta_{1} & \left.m_{2} g 1_{2} \sin \theta_{2}\right]^{T}
\end{array}\right.
\end{gathered}
$$

where, $g$ is the gravity effect. The state vector $\mathrm{q}$ and the control vector $\bar{\tau}$ are defined as: 
Int. J. Syst. Signal Control Eng. Appl., 5 (1): 5-13, 2012

$$
\begin{aligned}
& q=\left[\begin{array}{lll}
x & \theta_{1} & \theta_{2}
\end{array}\right]^{\mathrm{T}} \\
& \bar{\tau}=\left[\begin{array}{lll}
F & 0 & 0
\end{array}\right]^{\mathrm{T}}
\end{aligned}
$$

After rearranging Eq. 1 and multiplying both sides by $\mathrm{M}^{-1}$, one obtains:

$$
\ddot{\mathrm{q}}=\mathrm{M}^{-1}(-\mathrm{C} \dot{\mathrm{q}}-\mathrm{G}+\bar{\tau})
$$

Where $\mathrm{M}^{-1}$ is guaranteed to exist due to $\operatorname{det}(\mathrm{M})>0$. In this study, the values of the parameters (Liu et al., 2006) are defined as: $m=5 \mathrm{~kg}, \mathrm{~m}_{1}=2 \mathrm{~kg}, \mathrm{~m}_{2}=5 \mathrm{~kg}, 1_{1}=2 \mathrm{~m}$, $1_{2}=1 \mathrm{~m}$ and $\mathrm{g}=9.8 \mathrm{~m} \mathrm{sec}^{-2}$.

In this study, control schemes for rigid body motion control of the cart and swaying angle reduction of double pendulum are proposed. Initially, the SIFLC controller is designed. Then, both positive and negative input shapers are incorporated in the closed-loop system for control of both hook and load sway angle.

Fuzzy Logic Controller (FLC) is a linguistic-based controller that tries to emulate the way human thinking in solving a particular problem by means of rule inferences. Typically, a FLC has two controlled inputs, namely error (e) and the change of error (e). Its rule table can be created on a two-dimensional space of the phase-plane (e, e) as shown in Table 1. It is common for the rule table to have the same output membership in a diagonal direction.

Additionally, each point on the particular diagonal lines has a magnitude that is proportional to the distance from its main diagonal line $\mathrm{L}_{z}$. This is known as the Toeplitz structure.

The Toeplitz property is true for all FLC types which use the error and its derivative terms, namely e,è and $\mathrm{e}^{(\mathrm{n}-1)}$ as input variables (Ayob et al., 2009). By observing the consistent patterns of the output memberships in Table 1, there is an opportunity to simplify the table considerably.

Instead of using two-variable input sets (e,e), it is possible to obtain the corresponding output, $\mathrm{u}_{0}$ using a single variable input only. The significance of the reduction was first realised by Choi et al. (2000) and is known as the Signed Distance Method. The method simplifies the number of inputs into a single input variable known as distance, $\mathrm{d}$.

The distance represents the absolute distance magnitude of the parallel diagonal lines (in which the input set of $\mathrm{e}$ and $\dot{e}$ lies) from the main diagonal line

\begin{tabular}{|c|c|c|c|c|c|c|c|}
\hline \multirow[b]{2}{*}{$\dot{\mathrm{e}}$} & \multicolumn{7}{|l|}{ e } \\
\hline & PL & PM & PS & Z & NS & $\mathrm{NM}$ & $\mathrm{NL}$ \\
\hline $\mathrm{NL}$ & $\mathrm{Z}$ & NS & $\mathrm{NM}$ & $\mathrm{NL}$ & $\mathrm{NL}$ & $\mathrm{NL}$ & $\mathrm{NL}$ \\
\hline $\mathrm{NM}$ & PS & Z & NS & $\mathrm{NM}$ & NL & NL & $\mathrm{NL}$ \\
\hline NS & PM & PS & Z & NS & $\mathrm{NM}$ & NL & $\mathrm{NL}$ \\
\hline Z & PL & PM & PS & Z & NS & $\mathrm{NM}$ & $\mathrm{NL}$ \\
\hline PS & PL & PL & PM & PS & Z & NS & $\mathrm{NM}$ \\
\hline PM & PL & PL & PL & PM & PS & $\mathrm{Z}$ & NS \\
\hline PL & PL & PL & PL & $\mathrm{PL}$ & PM & PS & $\mathrm{Z}$ \\
\hline
\end{tabular}
$\mathrm{L}_{z}$. To derive the distance, $\mathrm{d}$ variable, let $\mathrm{Q}\left(\mathrm{e}_{0}, \dot{\mathrm{e}}_{0}\right)$
Table 1: Rule table with Toeplitz structure

Table 2: The reduced rule table using the Signed Distance Method

\begin{tabular}{llllllll}
\hline $\mathrm{d}$ & $\mathrm{L}_{\mathrm{NL}}$ & $\mathrm{L}_{\mathrm{NM}}$ & $\mathrm{L}_{\mathrm{NS}}$ & $\mathrm{L}_{Z}$ & $\mathrm{~L}_{\mathrm{PS}}$ & $\mathrm{L}_{\mathrm{PM}}$ & $\mathrm{L}_{\mathrm{PL}}$ \\
\hline $\mathrm{u}_{0}$ & $\mathrm{NL}$ & $\mathrm{NM}$ & $\mathrm{NS}$ & $\mathrm{Z}$ & $\mathrm{PS}$ & $\mathrm{PM}$ & $\mathrm{PL}$ \\
\hline
\end{tabular}

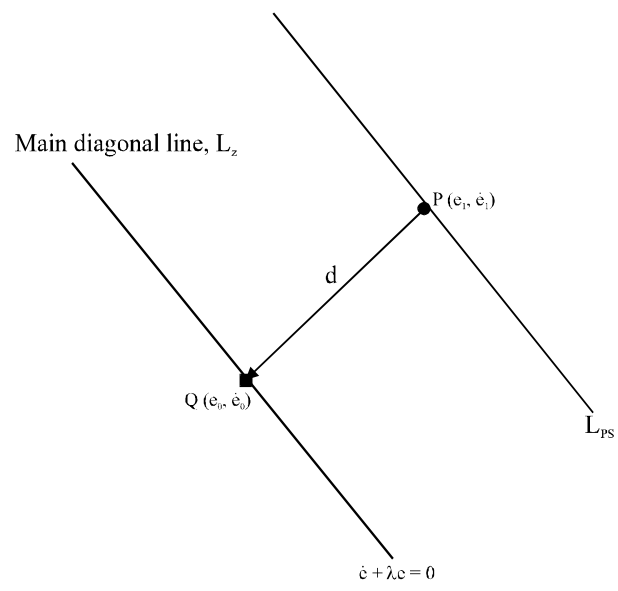

Fig. 2: Derivation of distance variable

be an intersection point of the main diagonal line and the line perpendicular to it from a known operating point $\mathrm{P}\left(\mathrm{e}_{1}, \mathrm{e}_{1}\right)$ as shown in Fig. 2. It can be noted that the main diagonal line can be represented as a straight line function, i.e.:

$$
\dot{\mathrm{e}}+\lambda \mathrm{e}=0
$$

In Eq. 6 , variable $\lambda$ is the slope magnitude of the main diagonal line $\mathrm{L}_{z}$. The distance $\mathrm{d}$ from point $\mathrm{P}\left(\mathrm{e}_{1}, \dot{\mathrm{e}}_{1}\right)$ to point $\mathrm{Q}\left(\mathrm{e}_{0}, \dot{\mathrm{e}}_{0}\right)$ can be obtained as (Ayob et al., 2009):

$$
\mathrm{d}=\frac{\dot{\mathrm{e}}+\lambda \mathrm{e}}{\sqrt{1+\lambda^{2}}}
$$

The derivation of distance input variable resulted in a one-dimensional rule table, in contrast to a twodimension table required by the conventional FLC. The reduced rule table is shown in Table 2 where $\mathrm{L}_{\mathrm{NL}}, \mathrm{L}_{\mathrm{NM}} \mathrm{L}_{\mathrm{NS}}$, $\mathrm{L}_{z}$, LPS $\mathrm{L}_{\mathrm{PM}}$ and $\mathrm{L}_{\mathrm{PL}}$ are the diagonal lines of Table 2 . The diagonal lines correspond to the new input of this rule table while NL, NM, NS, Z, PS, PM and PL represent the output of corresponding diagonal lines. As can be 
realized, the control action of FLC is now exclusively determined by $\mathrm{d}$. It is therefore appropriate to called it the Single Input FLC (SIFLC).

The overall structure of SIFLC derived from the Signed Distance Method can be shown in Fig. 3. Two system state variables e (position error) and $\mathrm{x}$ (velocity of the cart) are selected as the feedback signal. The input to the FLC block is the distance variable $\mathrm{d}$ while the output from FLC block is the change of control output $\mathrm{u}_{0}$. The final output of this FLC is obtained by multiplying $\mathrm{u}_{0}$ with the output scaling factor denoted as $r$. The output equation can be written as:

$$
\mathrm{u}=\dot{\mathrm{u}}_{0} \mathrm{r}
$$

Accordingly, the slope magnitude, $\lambda$ and output scaling factor, $\mathrm{r}$ for cart trajectory tracking were deduced as -1 and 56.03 , respectively. Next, the designed SIFLC is combined with input shaping scheme for control of rigid body motion of the cart and swaying angle reduction of the system. A block diagram of SIFLC with input shaping is shown in Fig. 4. Input shaping technique is a feed-forward control technique that involves convolving a desired command with a sequence of impulses known as input shaper.

The shaped command that results from the convolution is then used to drive the system. Design objectives are to determine the amplitude and time locations of the impulses so that the shaped command reduces the detrimental effects of system flexibility. These parameters are obtained from the natural frequencies of hook and load sway angle and damping ratios of the system. For the case of positive amplitudes, each

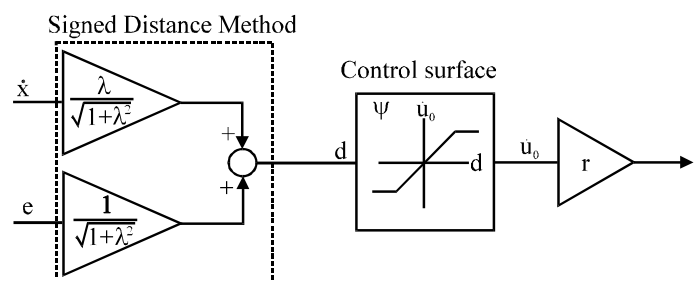

Fig. 3: SIFLC structure for DPTOC with linear control surface

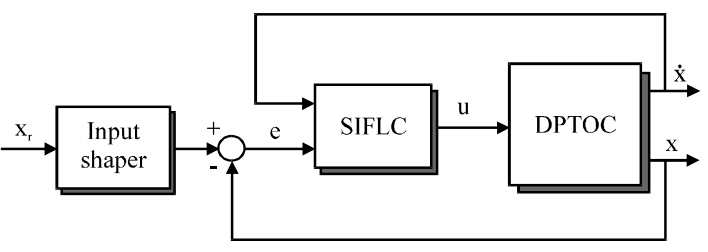

Fig. 4: SIFLC with input shaping control structure individual impulse must be $<1$ to satisfy the unity magnitude constraint. In order to increase the robustness of the input shaper to errors in natural frequencies, the positive Zero-Sway-Derivative-Derivative (ZSDD) input shaper (Ahmad et al., 2009a, d) is designed by solving the derivatives of the system sway equation. This yields a four-impulse sequence with parameter as:

$$
\begin{gathered}
\mathrm{t}_{1}=0, \mathrm{t}_{2}=\frac{\pi}{\omega_{\mathrm{d}}}, \mathrm{t}_{3}=\frac{2 \pi}{\omega_{\mathrm{d}}}, \mathrm{t}_{4}=\frac{3 \pi}{\omega_{\mathrm{d}}} \\
\mathrm{A}_{1}=\frac{1}{1+3 \mathrm{~K}+3 \mathrm{~K}^{2}+\mathrm{K}^{3}}, \mathrm{~A}_{2}=\frac{3 \mathrm{~K}}{1+3 \mathrm{~K}+3 \mathrm{~K}^{2}+\mathrm{K}^{3}} \\
\mathrm{~A}_{3}=\frac{3 \mathrm{~K}^{2}}{1+3 \mathrm{~K}+3 \mathrm{~K}^{2}+\mathrm{K}^{3}}, \mathrm{~A}_{4}=\frac{\mathrm{K}^{3}}{1+3 \mathrm{~K}+3 \mathrm{~K}^{2}+\mathrm{K}^{3}} \\
\mathrm{~K}=\mathrm{e}^{-\zeta \pi / \sqrt{1-\zeta^{2}}}, \omega_{\mathrm{d}}=\omega_{\mathrm{n}} \sqrt{1-\zeta^{2}}
\end{gathered}
$$

Where:

$\omega_{\mathrm{n}}$ and $\zeta=$ Representing the natural frequency and damping ratio, respectively

$\mathrm{t}_{\mathrm{j}}$ and $\mathrm{A}_{\mathrm{j}}=$ The time location and amplitude of impulse $\mathrm{j}$, respectively

The selection of natural frequency modes is very crucial in the performance of sway reduction. Input shaping techniques based on positive input shaper has been proved to be able to reduce sway of a system. In order to achieve higher robustness, the duration of the shaper is increased and thus, increases the delay in the system response. By allowing the shaper to contain negative impulses, the shaper duration can be shortened while satisfying the same robustness constraint. To include negative impulses in a shaper requires the impulse amplitudes to switch between 1 and -1 as:

$$
\mathrm{A}_{\mathrm{i}}=(-1)^{\mathrm{i}+1} ; \mathrm{i}=1,2, \ldots, \mathrm{n}
$$

The constraint in Eq. 10 yields useful shapers as they can be used with a wide variety of inputs without leading to over-currenting. For a Unity Magnitude (UM) negative Zero-Sway (ZS) shaper, i.e., the magnitude of each impulse is $|1|$, the shaper duration is one-third of the sway period of an undamped system while the shaper duration for the positive shaper is half of the sway period. In this research, the previous SNA input shaper (Mohamed et al., 2006) is modified by locating the negative amplitudes at the centre between each positive impulse sequences with even number of total impulses. This will result the shaper duration as one-fourth of the 
sway period of an undamped system as shown in Fig. 5. The modified SNA-ZSDD shaper is proposed and applied in this research to enhance the robustness capability of the controller while increasing the speed of the system response. By considering the form of modified SNA-ZSDD shaper shown in Fig. 5, the amplitude summation constraints equation can be obtained as:

$$
2 a+2 c-2 b-2 d=1
$$

The values of a-d can be set to any value that satisfy the constraint in Eq. 11. However, the suggested values of a-d are $<|1|$ to avoid the increase of the actuator effort.

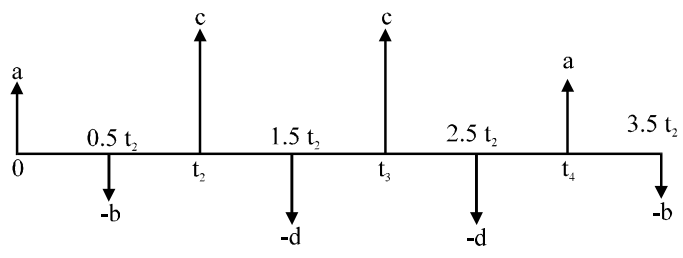

Fig. 5: Illustration of negative input shaping impulses
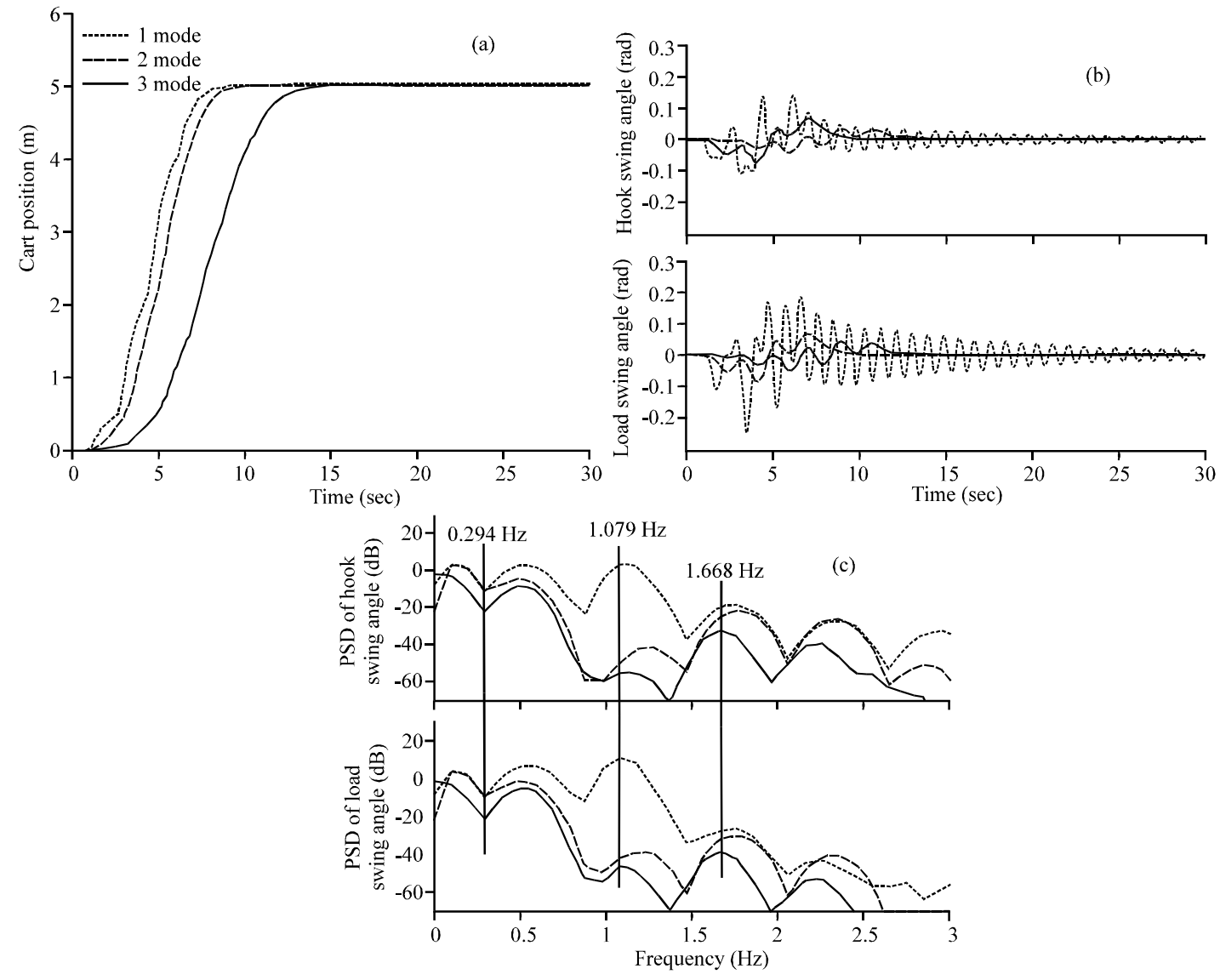

Fig. 6: Response of the DPTOC with hybrid SIFLC and positive input shaping; a) Cart position; b) Hook and load swing angle and c) PSD of hook and load swing angle 
Int. J. Syst. Signal Control Eng. Appl., 5 (1): 5-13, 2012
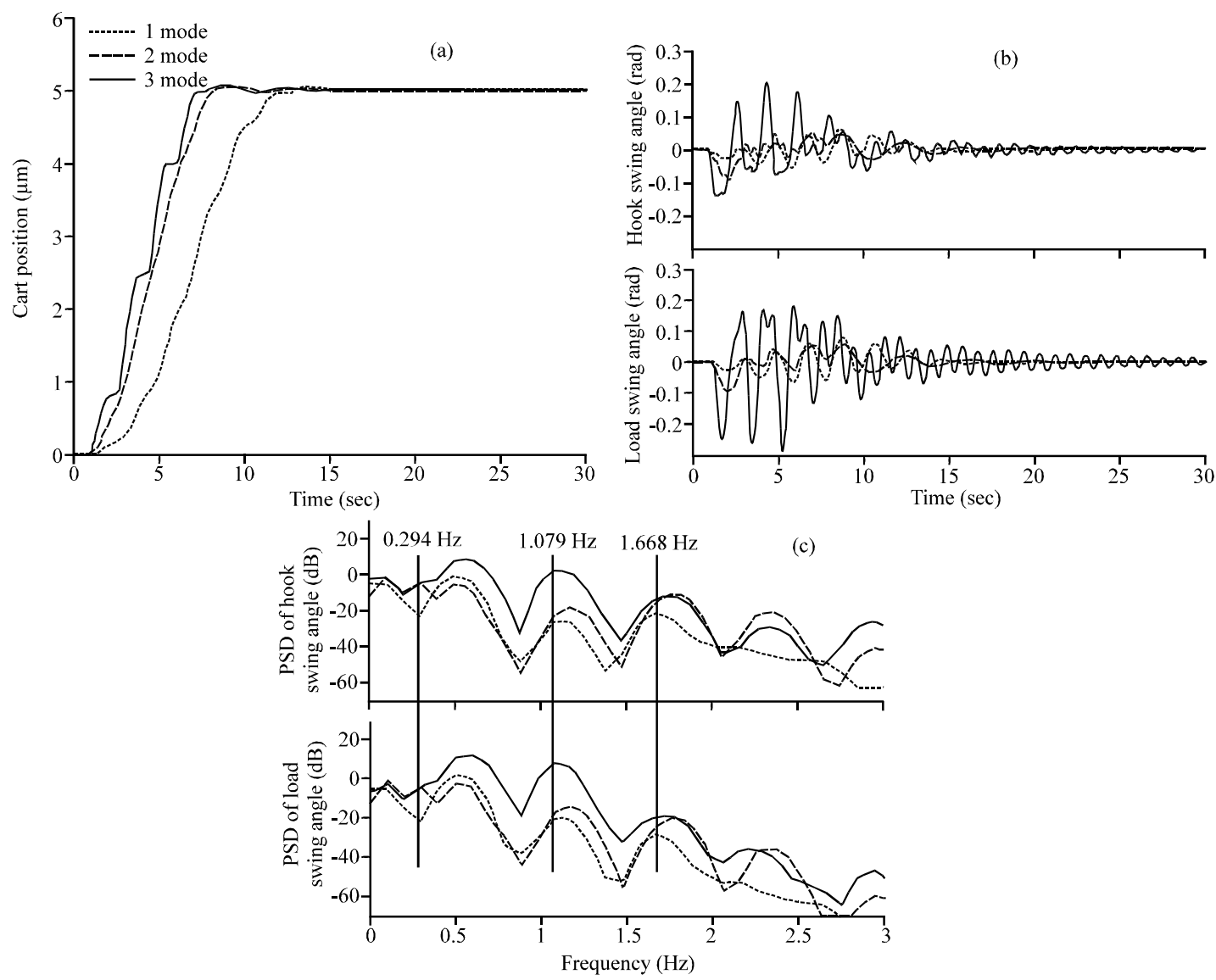

Fig. 7: Response of the DPTOC with hybrid SIFLC and negative input shaping; a) Cart position; b) Hook and load swing angle and c) PSD of hook and load swing angle

were considered as the system response to the unshaped input under tracking capability and will be used to evaluate the performance of the input shaping schemes with three types of mode combination. Implementation results with SIFLC controller have shown that the steady-state cart position trajectory of $5 \mathrm{~m}$ for the DPTOC System was achieved within the rise and settling times and overshoot of $1.979,5.354 \mathrm{sec}$ and $7.44 \%$, respectively. However, a noticeable amount of oscillation occurs during movement of the cart. It is noted from the sway of hook and load angle response with a maximum residual of \pm 0.6 and $\pm 0.8 \mathrm{rad}$, respectively. Moreover, from the PSD of both hook and load swing angle response, the sway frequency is dominated by the 1st three modes which is obtained as $0.294,1.079$ and $1.668 \mathrm{~Hz}$. The closed loop parameters with the SIFLC control will subsequently be used to design and evaluate the performance of hybrid controllers with input shapers.

The application of both positive and negative input shaper with single mode, 1st two and three modes of sway frequency are applied to the DPTOC System. With the natural frequency of $0.294,1.079$ and $1.668 \mathrm{~Hz}$, the time locations and amplitudes of the impulses for the proposed input shapers scheme were obtained by solving Eq. 9-11. However, the amplitudes of the modified SNA ZSDD shaper were deduced as $[0.3,-0.1,0.5,-0.2,0.5,-0.2,0.3$, $-0.1]$ while the time locations of the impulses were located at the half of the time locations of positive ZSDD shaper as shown in Fig. 5. The system responses of the DPTOC System to the shaped trajectory input using SIFLC with both positive and negative shapers are shown in Fig. 6 and 7 , respectively.

It is noted that the proposed control schemes are capable of reducing the system sway while maintaining the input tracking performance of the cart position. Similar cart position, hook and load swing angle and power spectral density of both hook and load swing angle responses were observed as compared to the SIFLC. Table 3 shows the level of sway reduction of the system responses at the different combination of modes in comparison to the SIFLC control. In overall, higher levels of sway reduction were obtained using SIFLC with three modes shaper as compared to the case with single and 
Int. J. Syst. Signal Control Eng. Appl., 5 (1): 5-13, 2012

Table 3: Level of sway reduction of the hook and load swing angle and specifications of cart position response

\begin{tabular}{|c|c|c|c|c|c|c|c|c|}
\hline \multirow[b]{2}{*}{ Types of shaper } & \multirow[b]{2}{*}{ Mode selection } & \multirow[b]{2}{*}{ Swing angle } & \multicolumn{3}{|c|}{$\begin{array}{l}\text { Attenuation }(\mathrm{dB}) \text { of } \\
\text { sway of the cable (mode) }\end{array}$} & \multicolumn{3}{|c|}{$\begin{array}{l}\text { Specification of cart } \\
\text { position response }\end{array}$} \\
\hline & & & & 2 & 3 & $\begin{array}{l}\text { Rise } \\
\text { time (sec) }\end{array}$ & $\begin{array}{l}\text { Settling } \\
\text { time (sec) }\end{array}$ & Overshoot (\%) \\
\hline \multirow[t]{6}{*}{ Positive } & 1 & Hook swing angle $\left(\theta_{1}\right)$ & 32.54 & 0.25 & -4.89 & 3.958 & 6.192 & 0.12 \\
\hline & & Load swing angle $\left(\theta_{2}\right)$ & 32.90 & 0.83 & 12.25 & & & \\
\hline & 2 & Hook swing angle $\left(\theta_{1}\right)$ & 31.49 & 53.19 & 0.19 & 4.315 & 6.975 & 0.02 \\
\hline & & Load swing angle $\left(\theta_{2}\right)$ & 31.85 & 53.05 & 16.95 & & & \\
\hline & 3 & Hook swing angle $\left(\theta_{1}\right)$ & 43.52 & 58.28 & 7.48 & 6.048 & 10.780 & 0.00 \\
\hline & & Load swing angle $\left(\theta_{2}\right)$ & 43.46 & 57.97 & 23.78 & & & \\
\hline \multirow[t]{6}{*}{ Negative } & 1 & Hook swing angle $\left(\theta_{1}\right)$ & 25.26 & 1.03 & -11.39 & 4.983 & 6.013 & 1.24 \\
\hline & & Load swing angle $\left(\theta_{2}\right)$ & 25.32 & 1.91 & 2.91 & & & \\
\hline & 2 & Hook swing angle $\left(\theta_{1}\right)$ & 25.51 & 26.55 & -9.54 & 4.958 & 6.857 & 0.96 \\
\hline & & Load swing angle $\left(\theta_{2}\right)$ & 25.86 & 27.98 & 7.52 & & & \\
\hline & 3 & Hook swing angle $\left(\theta_{1}\right)$ & 44.48 & 30.19 & -3.78 & 6.515 & 10.510 & 0.96 \\
\hline & & Load swing angle $\left(\theta_{2}\right)$ & 43.54 & 30.82 & 12.47 & & & \\
\hline
\end{tabular}

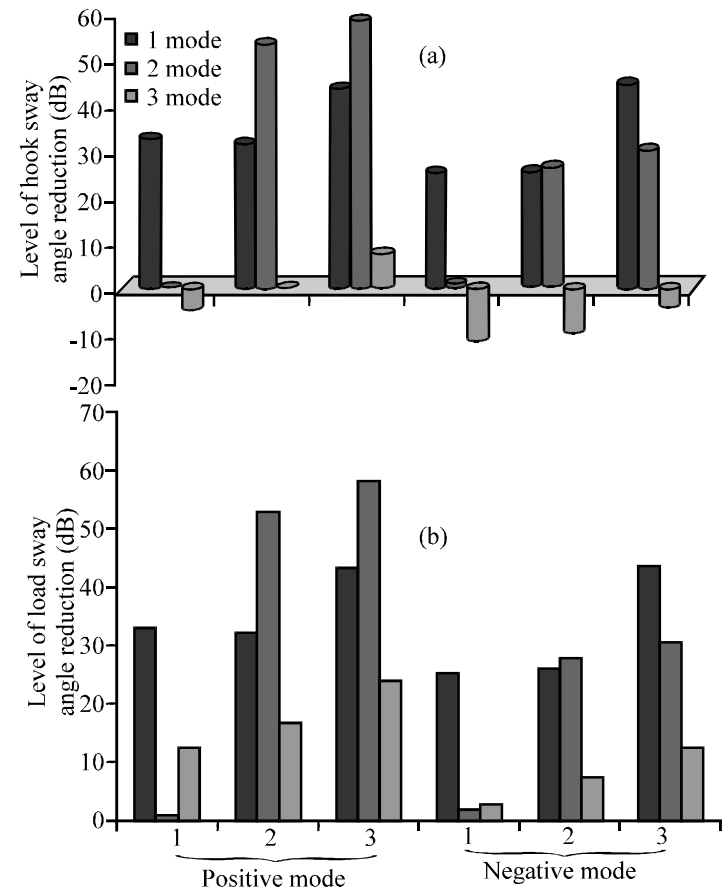

Fig. 8: Level of hook and load swing angle reduction; a) Hook swing angle; b) Load swing angle

two modes shaper. This can be clearly observed from the response of both hook and load sway angle in Fig. $6 \mathrm{~b}$ and $7 \mathrm{~b}$ for positive and negative input shaping, respectively. However with single mode shaper, the cart position response as shown in Fig. 6a and 7a is faster as compared to higher number of mode combinations. It shows that for both positive and negative input shaping, the speed of the system response reduces with the increase in number of modes as well as number of impulse sequence. The corresponding rise time, setting time and overshoot of the cart position response using SIFLC control with single mode, 1 st two and three modes of positive and negative shapers is shown in Table 3 . It is also noted that a slower cart position response for SIFLC

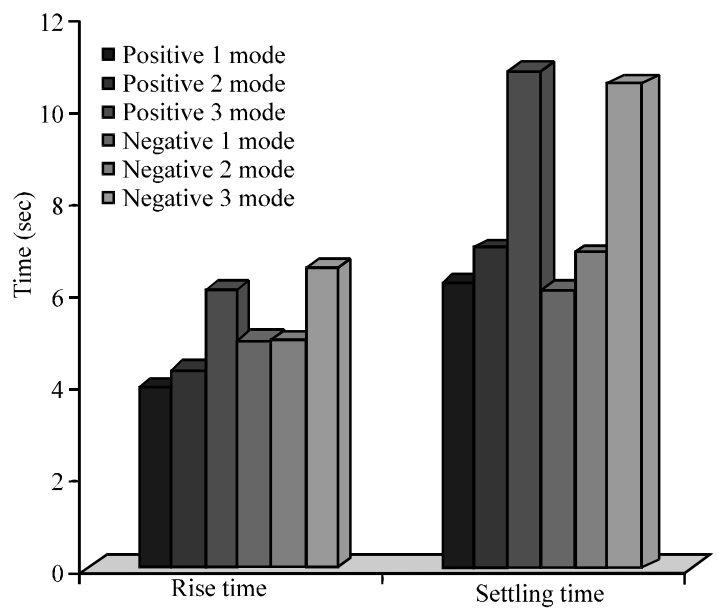

Fig. 9: Rise and settling time of cart position response

with input shaping control schemes as compared to the SIFLC control was achieved. Moreover, the overshoot of the cart position response is also reduced with the increase in number of modes.

Besides, comparison in different polarity input shaping also can be shown in Table 3 . In general, it is noted that the higher performance in the reduction of sway of the system is achieved using positive input shaping. This is observed and compared to the negative input shaping at the single mode, 1 st two and three modes of sway frequency. For comparative assessment, the levels of hook and load sway reduction using both positive and negative shapers are shown with the bar graphs in Fig. 8 .

The result shows that for both hook and load sway angle, the highest level of sway reduction is achieved in control schemes using the positive shaper with all combination of sway frequency modes. Therefore, it can be concluded that the positive ZSDD shapers provide better performance in sway reduction as compared to the 
Int. J. Syst. Signal Control Eng. Appl., 5 (1): 5-13, 2012

modified SNA ZSDD shapers in overall. Comparisons of the time response specifications of the cart position responses of input shaping control schemes using both positive and negative shapers are shown in Fig. 9 for the rise and settling times. It is noted that the settling time of the cart position response by using the modified SNA ZSDD shaper is smaller than the case using the positive ZSDD shaper. It shows that the speed of the system response can be improved by using a negative impulse input shapers.

\section{CONCLUSION}

The development of hybrid control schemes based on SIFLC control with positive and negative shapers in single mode, first two and three modes for input tracking and sway suppression of a DPTOC System has been presented. The performances of the control schemes have been evaluated in terms of input tracking capability, level of sway reduction and time response specifications. Acceptable performance in input tracking control and sway suppression has been achieved with proposed control strategies. Moreover, a significant reduction in the system sway has been achieved with the hybrid controllers regardless of the number of modes in the input shapers design. A comparison of the results has demonstrated that the SIFLC control with higher number of input shaper modes provide higher level of sway reduction as compared to the cases using lower number modes for both positive and negative shapers. However with lower number of modes, the speed of the response is slightly improved at the expenses of decrease in the level of sway reduction. Comparative assessment also demonstrated that the positive shapers provide higher level of sway reduction as compared to the cases using modified negative SNA shapers.

By using the modified negative input shapers, the speed of the response is slightly improved in term of settling time at the expenses of decrease in the level of sway reduction. It is concluded that the proposed hybrid controllers are capable of reducing the system sway while maintaining the input tracking performance of the DPTOC.

\section{ACKNOWLEDGEMENTS}

This research was supported by Faculty of Electrical and Electronics Engineering, Universiti Malaysia Pahang, especially Control and Instrumentation (COINS) Research Group and Ministry of Science, Technology and Innovation (MOSTI) under Fundamental Research Grant Scheme RDU100102.

\section{REFERENCES}

Ahmad, M.A., R.M.T. Raja Ismail and M.S. Ramli, 2009a. Hybrid input shaping and non-collocated PID control of a gantry crane system: Comparative assessment. Proceedings of IEEE/ASME International Conference on Advanced Intelligent Mechatronics, July 14-17, 2009, Singapore, pp: 1792-1797.

Ahmad, M.A., R.M.T. Raja Ismail and M.S. Ramli, $2009 \mathrm{~b}$. Input shaping techniques for anti-sway control of a 3-D gantry crane system. Proceedings of the IEEE International Conference on Mechatronics and Automation, August 9-12, 2009, Changchun, China, pp: $2876-2881$.

Ahmad, M.A., R.M.T. Raja Ismail, M.S. Ramli, N.F. Zakaria and N.M. Abd Ghani, 2009c. Robust feed-forward schemes for anti-sway control of rotary crane. Proceedings of the International Conference on Computational Intelligence, Modelling and Simulation, Septtember 7-9, 2009, IEEE Computer Society, Washington, DC, USA., pp: 17-22.

Ahmad, M.A., R.M.T. Raja Ismail, M.S. Ramli, N.M.A. Ghani and N. Hambali, 2009d. Investigations of feed-forward techniques for anti-sway control of 3-D gantry crane system. IEEE Symp. Indus. Electron. Appl., 1: 265-270.

Ahmad, M.A., R.M.T.R. Ismail, M.S. Ramli, A.N.K. Nasir and N. Hambali, 2009e. Feed-forward techniques for sway suppression in a double-pendulum-type overhead crane. Int. Conf. Comp. Technol. Dev., 1: 173-178.

Ahmad, M.A., Z. Zulkifely and M.A. Zawawi, 2010. Experimental investigations of input shaping schemes for sway control of a gantry crane system. Proceedings of the 2nd International Conference on Computer and Network Technology, April 23-25, 2010, Bangkok, Thailand, pp: 483-486.

Auernig, J.W. and H. Troger, 1987. Time optimal control of overhead cranes with hoisting of the load. Automatica, 23: 437-447.

Ayob, S.M., N.A. Azli and Z. Salam, 2009. PWM DC-AC converter regulation using a multi-loop single input fuzzy PI controller. J. Power Electron., 9: 124-131.

Choi, B.J., S.W. Kwak and B.K. Kim, 2000. Design and stability analysis of single-input fuzzy logic controller. IEEE Trans. Syst. Man Cybern., 30: 303-309.

Lee, H.H. and S.K. Cho, 2001. A New fuzzy logic anti-swing control for industrial three-dimensional overhead crane. Proceedings of the IEEE International Conference on Robotic and Automation, May 21-26, 2001, COEX. Seoul, Korea, pp: 2958-2961. 
Liu, D.T., W.P. Guo and J.Q. Yi, 2006. Dynamics and stable control for a class of underactuated mechanical systems. Acta Automatica Sinica, 32: 422-427.

Manson, G.A., 1992. Time-optimal control of and overhead crane model. Optimal Control Appl. Methods, 3: 115-120.

Mohamed, Z., A.K. Chee, A.W.I. Mohd Hashim, M.O. Tokhi, S.H.M. Amin and R. Mamat, 2006. Techniques for vibration control of a flexible manipulator. Robotica, 24: 499-511.
Omar, H.M., 2003. Control of gantry and tower cranes. Ph.D. Thesis, Virginia Polytechnic Institute and State University, Blacksburg, Virginia.

Spong, M.W., 1998. Underactuated Mechanical Systems. In: Control Problems in Robotics and Automation, Siciliano, B. and K. Valavanis (Eds.). Springer-Verlag, London, UK., pp: 135-150.

Yang, J.H. and K.S. Yang, 2007. Adaptive coupling control for overhead crane systems. Mechatronics, 17: $143-152$ 